\title{
Retrograde Renal Arteriography Using a Balloon-fitted Vascular Catheter
}

\author{
By \\ Sentaro Shishito, Atsuo Sugita and Yutaka Onodera \\ From the Department of Urology, Tohoku University School of \\ Medicine, Sendai
}

(Received for publication, November 19, 1964)

\begin{abstract}
Of various renal arteriographic methods, selective renal arteriography is the most excellent to produce distinct renal arteriograms. This method is, however, technically so complicated, and requires a long time and cannot visualize both the right and left renal arteries. Therefore, we employed a balloon-fastened catheter and succeeded in visualizing both the renal arteries at the same time by taking a radiograph, while the blood stream running to the lower extremities was blocked at the bifurcation of common iliac arteries with the balloon. This ballooncatheter method is technically simple and needs no fluoroscopic examination for determining where to place the catheter. In addition, it can be done in a comparatively short time. We, therefore, present here the technique and effects of this radiographic method. In addition, we describe in detail the findings on the cases we examined by this renal arteriography and discuss its clinical value. We would like here to recommend the ballooncatheter method especially for visualization of Goldblatt type renal arterial stenosis, for it can distinctly visualize the lesion.
\end{abstract}

Abdominal aortography can visualize the branches of the abdominal aorta, particularly the renal arteries. Its clinical value is, therefore, highly estimated in the differential diagnosis and treatment of various renal diseases. This radiographic technique is classified into 4 kinds of radiography, according to the method of injection of a contrast medium: 'Translumbar abdominal aortography, retrograde abdominal aortography, selective renal arteriography and intravenous abdominal aortography. Among them, the selective renal arteriography produces the most definite renal arteriogram. This method is, however, much complicated, requires a long time, and has a disadvantage of being unable to visualize both the right and left renal arteries at the same time. While looking for a method which can produce pictures visualizing both the right and left renal arteries in a short time as distinctly as in the selective method, we happened to know that Watanabe ${ }^{1}$ in his radiographic examination, employed a two-cavity intracardiac catheter equipped with a balloon, the blood stream being interrupted from running toward the 
lower extremities. We improved his technique and succeeded in obtaining so clear renal arteriograms that we could almost attain our purpose. This radiographic technique which we improved is introduced in this paper, and the results of the synthetic observations of the cases examined are also reported.

\section{1) Instrument}

\section{METHODS}

Two vascular catheters were employed in combination. A No. 7 vascular catheter was fitted at its end with a thin rubber balloon capable of carrying 20 c.c. (Fig. 1) and then fastened to a No. 9-11 vascular catheter at $5 \mathrm{~cm}$ from the tip. This combined catheter was connected with a 50 c.c. injection syringe of LuerLock type which was installed with a 2 -way cock.

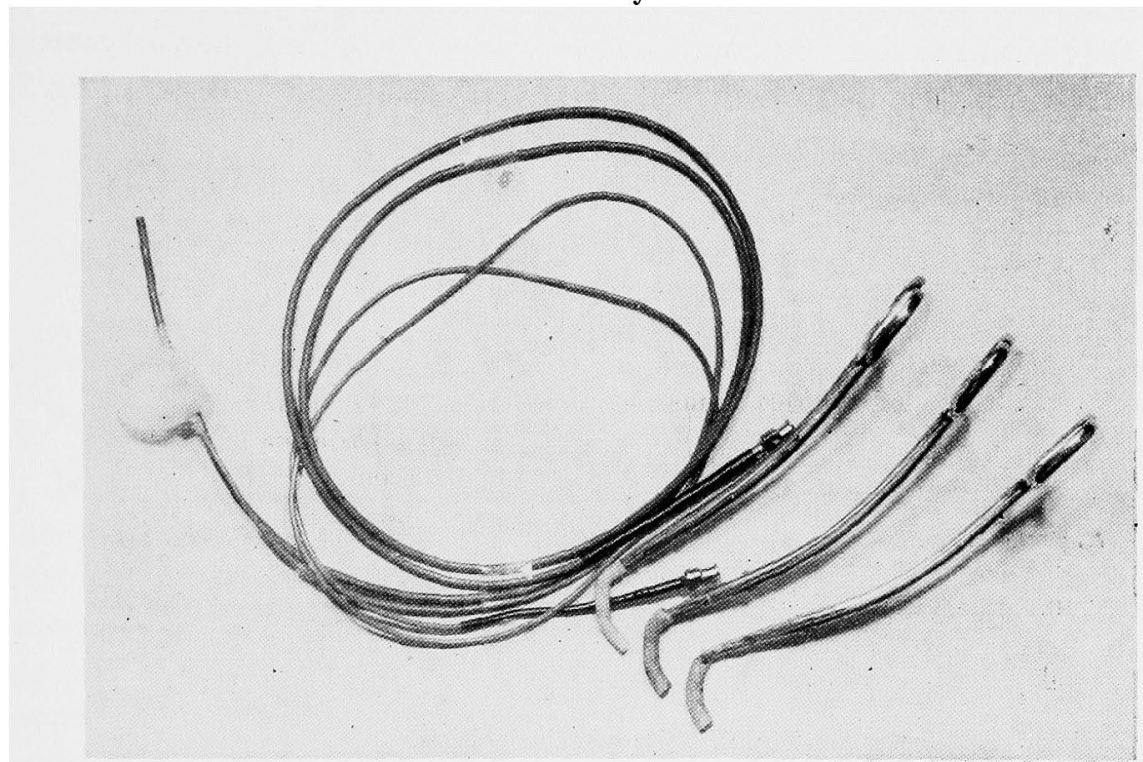

Fig. 1. A balloon-fitted catheter which we devised: A balloon is fastened to the end of a No. 7 vascular catheter, which is then fixed to a No. 11 vascular catheter at $5 \mathrm{~cm}$ from the latter's end.

\section{2) Contrast medium}

The contrast medium to be employed in this examination was required to be low in viscosity, toxicity and irritability and yet strong enough in visualizing effect. A number of organic jodized preparations which could satisfy the above requirements to some extent were available: for instance, there were sodium iodomethamate (Neo-Iopax), iodopyracet (Per-Abrodil, Pyraceton, Diodrast, Umbradil etc.) and sodium acetrizoate (Urokon, Triodan, Urokolin, Urokolin M, Urografin, Hypaque, etc.). The comparative study of these contrast media in renal 
arteriography was already made by Melick et al., ${ }^{2}$ who stated that $70 \%$ Urokon was the most a vailable medium for that purpose. We examined various contrast media and found that $76 \%$ Urografin (Schering), a derivative of sodium acetrizoate, has less irritability and side-effects than $70 \%$ Urokon. Accordingly, we employed $76 \%$ Urografin in a dosage of 10 to 20 c.c. in the following examination.

3) Exposure factors

The picture was taken at 70-80 KVP (secondary voltage), 150-200 mA, 0.10.2 seconds and $80 \mathrm{~cm}$ (film-focus) distance. A lysholm blende and intensifying screen were employed.

\section{4) Technique}

Local infiltration anesthesia was first carried out over the area of about $10 \mathrm{~cm}$ along the right femoral artery from 1 finger-breadth below the inguinal ligament, and then an incision of about $7 \mathrm{~cm}$ long was given to the skin of this area (Fig. 2).

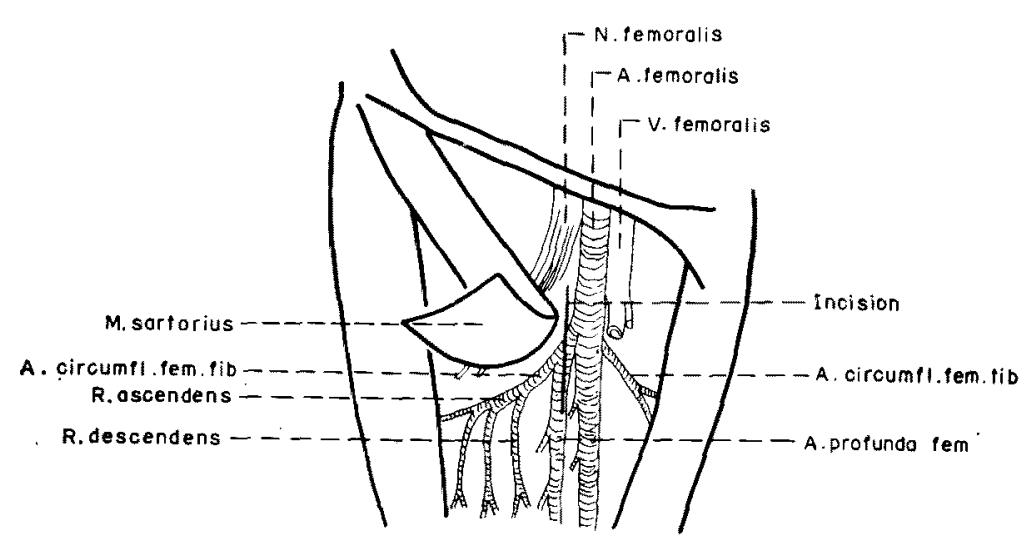

Fig. 2. Site of skin incision.

When the sartorius muscle was reached, the femoral artery was exposed out by pushing it outside. In the next place, pushing the femoral artery inward revealed out the deep femoral artery, which was then completely peeled out about $3 \mathrm{~cm}$ long. After that, cotton tapes were placed above and below the peeled artery and a Satinsky clamp was used to block the blood stream. Then a longitudinal incision is given $1 \mathrm{~cm}$ long to the anterior wall of the deep femoral artery, though which the balloon-fitted catheter is inserted (Fig. 3). In the next place, 10 c.c. of a physiologic saline solution was injected into the balloon, inflation of which lead to the interruption of the blood stream toward the lower extremities. Then, the picture was taken, while $10-15$ c.c. of $76 \%$ Urografin was being injected in 1-1.5 seconds. After the completion of the radiographic examination, the 


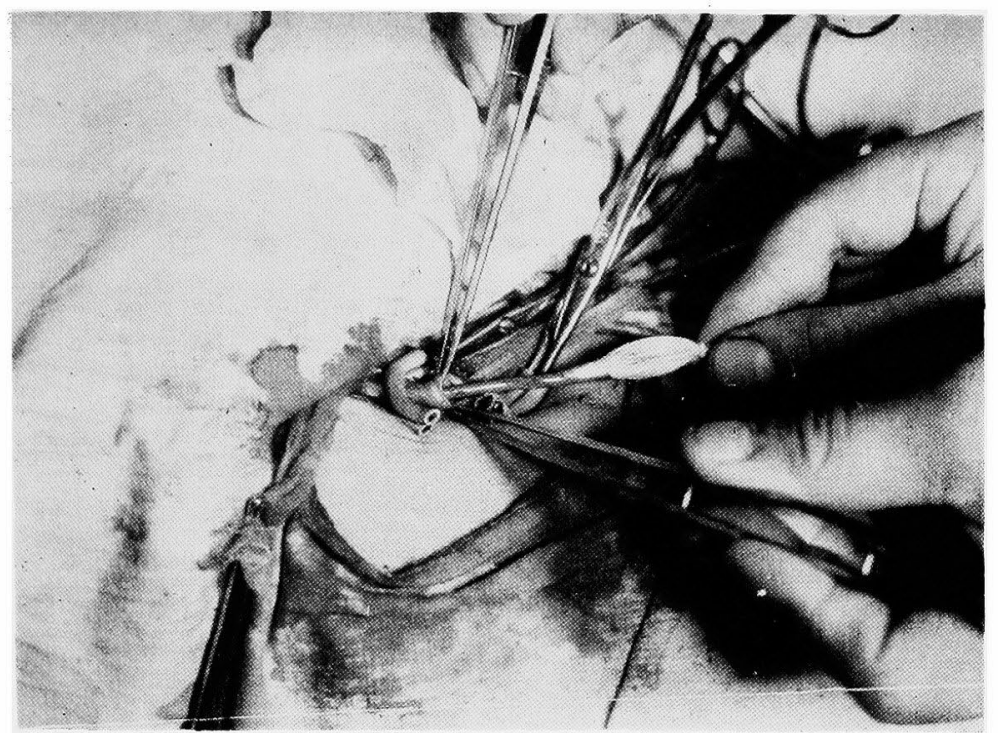

Fig. 3. The balloon-fitted catheter is being inserted into the deep femoral artery.

catheter was taken off and the incised portion of the vessel was then sutured.

\section{5) Factors in obtaining a distinct renal arteriogram}

When the balloon was inflated inside the aorta, interrupting the blood stream to the lower extremities, even if it had been immediately below the ramification of the renal artery, the balloon was observed to be pushed down by the aortic pressure as far as the ramification of the common iliac arteries. Therefore, we examined how far the tip of the catheter should go above the ramification of the common iliac arteries in order to obtain a distinct renal arteriogram, when the blood stream to the lower extremities was to be blocked at the bifurcation of the

TABLE I. Location of the Catheter and Distinctness of the Obtained Pictures in Retrograde Abdominal Aortograph (Using a Balloon)

\begin{tabular}{c|c|c|c|c}
\hline \multirow{2}{*}{ Distinctness } & $\begin{array}{c}\text { Location: distance from the ramifica- } \\
\text { tion of renal arteries in cm }\end{array}$ \\
& 1 & 2 & 3 & 4 \\
\hline I & 0 & 0 & 0 & 0 \\
II & 0 & 1 & 0 & 0 \\
III & 3 & 9 & 2 & 6 \\
IV & 0 & 1 & 8 & 4 \\
\hline Total (cases) & 3 & 11 & 10 & 10
\end{tabular}


common iliac arteries. As a result, it was found that the most distinct renal arteriogram could be obtained when the tip of the catheter was 3 to $4 \mathrm{~cm}$ below the ramification of the renal arteries (Table I). In addition, the distance from the ramification of the renal arteries to that of the common iliac arteries was examined by abdominal aortograms and demonstrated to be 8 to $12 \mathrm{~cm}$ in most cases. When the balloon was filled with 10 c.c. of a physiologic saline solution, it enlarged up to about $3 \mathrm{~cm}$ in diameter. It is, therefore, learned that a balloon has to be fixed at 3 to $6 \mathrm{~cm}$ from the tip of the catheter in order to obtain a distinct renal arteriogram. In fact, we employed the catheter with a balloon fixed at $5 \mathrm{~cm}$ from its tip.

\section{CLINICAL OBSERVATIONS OF RENAL ARTERIOGRAMS}

We performed renal arteriography in 174 cases during the period from April, 1959 to December, 1963. Of them, 140 cases were examined by the translumbar method, 22 eases by the retrograde method using a balloon and 12 cases by using the catheter alone. These 174 cases were classified according to the kinds of disease as in Table II.

TABLE II. Cases Examined by Renal Arteriography

\begin{tabular}{l|c}
\multicolumn{1}{c|}{ Diseases } & No. of cases \\
\hline Renal tuberculosis & 42 \\
Nephroptosis & 30 \\
Renal neoplasm & 24 \\
Essential hematuria & 21 \\
Hydronephrosis & 18 \\
Unilateral renal hypertension & 10 \\
Calculosis in the upper urinary tract & 7 \\
Congenital solitary kidney & 5 \\
Renal hypoplasia & 4 \\
Horse-shoe kidney & 3 \\
Renal cyst & 3 \\
Others & 7 \\
\hline Total & 174 \\
\hline
\end{tabular}

Number of the cases examined twice .......27

Number of the cases examined three times ... 8

\section{1) Normal renal arteriograms}

The blood vessels which can be successfully visualized by renal arteriography are not only the renal arteries, but also various arteries ramifying from the abdominal aorta such as the common hepatic artery, gastroduodenal artery, splenic artery, superior mesenteric artery and lumbar arteries. If a balloon was employed in radiography, however, selective visualization of both the renal arteries could be obtained (Fig. 4). 


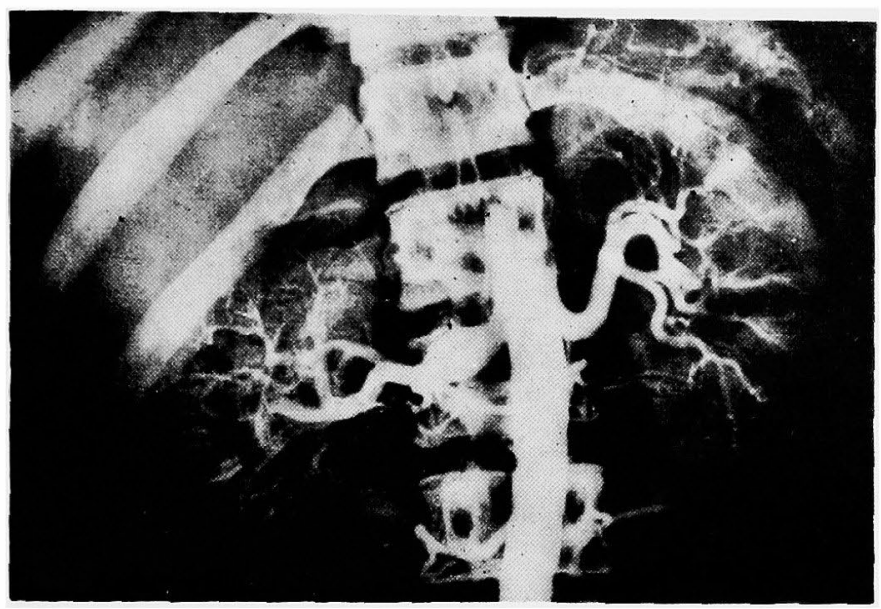

Fig. 4. A normal renal arteriogram (taken by employing a balloon catheter).

TABLE III. Inner Diameter of Normal Renal Artery

\begin{tabular}{|c|c|c|c|c|}
\hline \multicolumn{3}{|c|}{$\begin{array}{c}\text { Cases } \\
\text { (name, sex, age) }\end{array}$} & $\begin{array}{l}\text { Right renal } \\
\text { artery }(\mathrm{mm})\end{array}$ & $\begin{array}{l}\text { Left renal artery } \\
(\mathrm{mm})\end{array}$ \\
\hline T.K. & $\hat{\jmath}$ & 48 & 4.5 & 6.3 \\
\hline W.J. & p & 45 & 5.3 & 5.4 \\
\hline E.M. & $\delta$ & 32 & 5.4 & 6.3 \\
\hline A.F. & 우 & 39 & 4.5 & 6.3 \\
\hline S.H. & c & 38 & 5.4 & 5.4 \\
\hline H.T. & f & 29 & 3.6 & 5.4 \\
\hline K.I. & 웅 & 40 & 5.4 & 6.2 \\
\hline T.K. & 우 & 26 & 4.5 & 4.5 \\
\hline M.K. & 우 & 36 & 6.3 & 5.4 \\
\hline K.N. & 우 & 30 & 3.6 & 4.5 \\
\hline K.K. & $\hat{0}$ & 24 & 6.3 & 5.4 \\
\hline T.C. & $\hat{\sigma}$ & 18 & 6.3 & 6.3 \\
\hline O.T. & 우 & 44 & 5.4 & 5.4 \\
\hline S.K. & $\hat{b}$ & 22 & 5.4 & 5.4 \\
\hline U.T. & ธ & 22 & 6.3 & 5.4 \\
\hline S.H. & $\delta$ & 34 & 5.4 & 5.4 \\
\hline B.T. & $\delta$ & 43 & 6.3 & 5.4 \\
\hline A.I. & $\hat{\delta}$ & 31 & 6.3 & 7.2 \\
\hline G.K. & \& & 62 & 4.5 & 5.0 \\
\hline N.H. & 운 & 43 & 5.4 & 4.5 \\
\hline S.Y. & q & 56 & 5.4 & 6.2 \\
\hline Total & & 21 & 5.4 (average) & 5.6 (average) \\
\hline
\end{tabular}

In addition, we measured the caliber of the renal artery according to the method introduced by Maluf and $\mathrm{McCoy}^{3}$ and $\mathrm{Ohtani}^{4}$ in 21 cases on their normal renal arteriograms. The average caliber was proved to be $5.4 \mathrm{~mm}$ on the right side and $5.6 \mathrm{~mm}$ on the left side. This finding coincided with that reported by Ohtani (Table III). 


\section{2) Congenital solitary kidney}

This condition can often be diagnosed by pyelography or retroperitoneal pneumography, but these rediographic examinations cannot give accurate findings in some cases. Particularly in the cases where both the orifices of the ureters are cystoscopically observed and the ureters are found to remain still but incomplete, it is difficult to differentiate this condition from occlusive renal tuberculosis. On such occasion, renal arteriography proves itself to be an excellent unique method for giving an accurate diagnosis. We had such experience in 5 cases (Fig. 5).

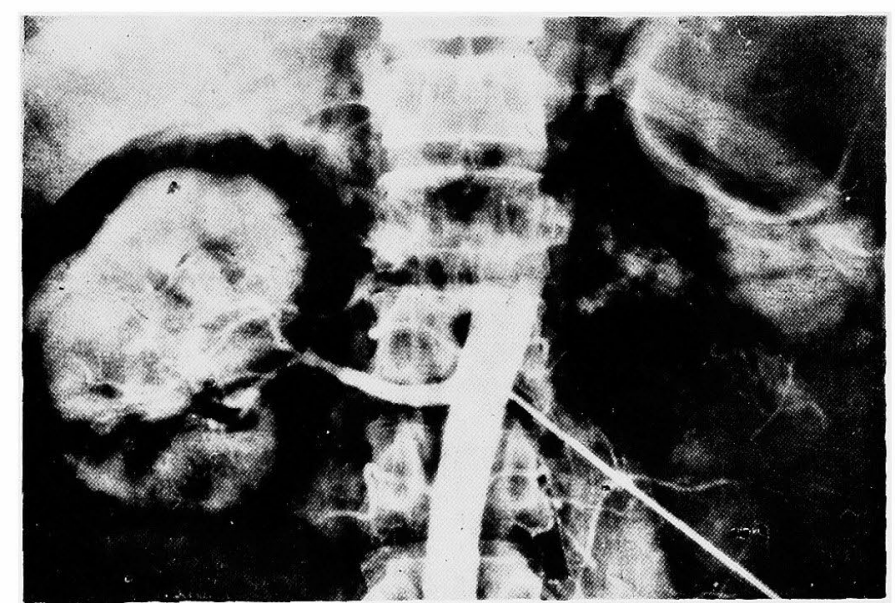

Fig. 5. Congenital solitary kidney combined retroperitoneal pneumography.

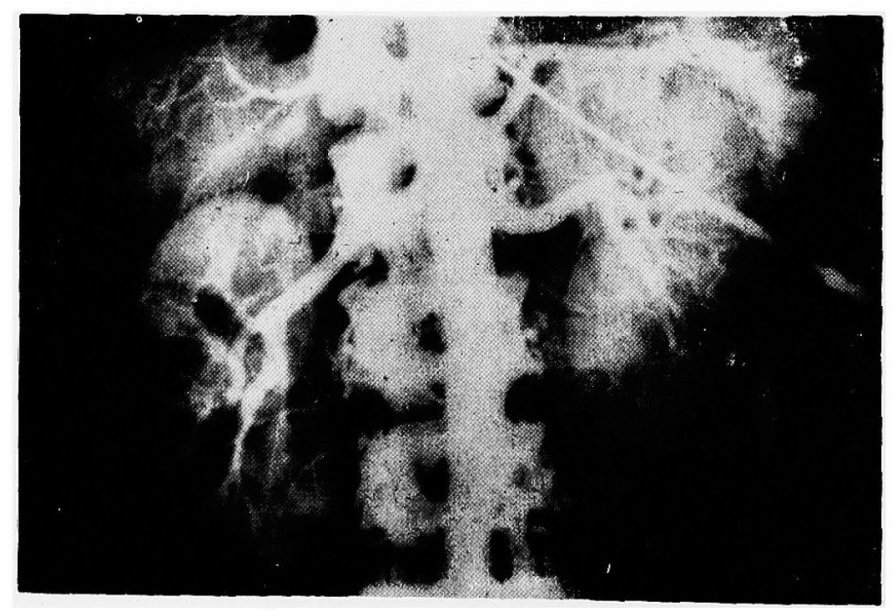

Fig. 6. Right nephroptosis. 


\section{3) Nephroptosis}

As renal arteriography discloses the length and the running state of the renal arteries, it can differentiate congenital ectopia from postnatal nephroptosis (Fig. 6). In addition, many cases of this condition are complicated by pyelonephritis. If this change diffuses, the caliber of the affected renal artery is narrowed and the intrarenal arterial branches become rougher and narrower, looking like an inelastic withered tree on a renal arteriogram, and a nephrogram turns out faint on the affected side. Such a finding was more markedly visualized if a balloon eatheter was employed in the examination (Fig. 7).

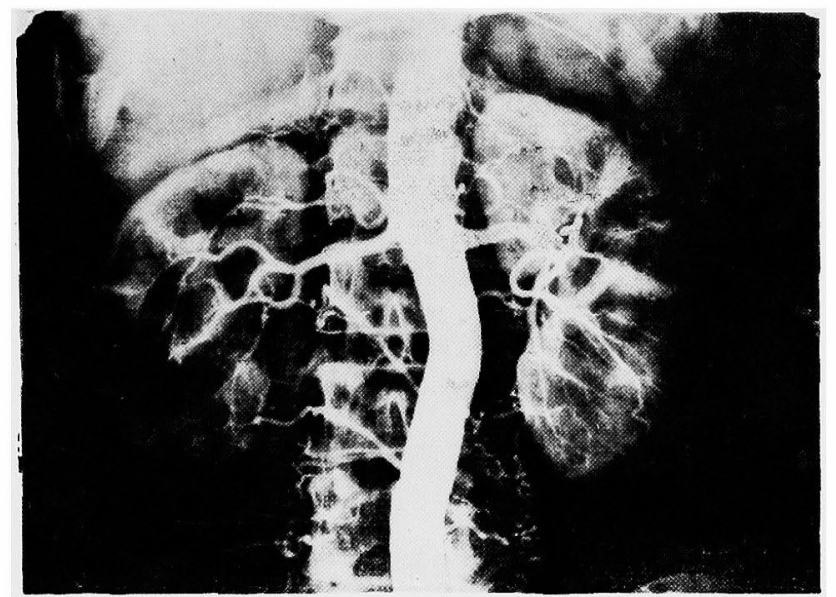

Fig. 7. Right nephroptosis combined with pyelonephritis (taken by employing a balloon catheter).

\section{4) Hydronephrosis}

In this condition, an intravenous pyelogram is sometimes unobtainable because of renal hypofunction, and retrograde pyelography cannot be utilized in blocked ureters. Furthermore, it results from aberrant vessels in some cases. On such occasions, renal arteriography is a unique diagnostic method, by which the presence of abnormal arteries, the dominating area of such abnormal arteries in the renal parenchyma and the residual of renal function can be brought to light. It has been already pointed out by Doss ${ }^{5}$ and Smith et al. ${ }^{6}$ that the renal function can be estimated from the findings of renal arteriography. According to them, reduction in the size of the renal artery and looseness of the intrarenal arterial branches give a diagnosis of hydronephrosis with renal function devastated. Therefore, we should emphasize the necessity of carrying out renal arteriography in all hydronephrotic patients who are going to undergo surgical treatment.

We carried out this radiographic examination in 18 patients with 


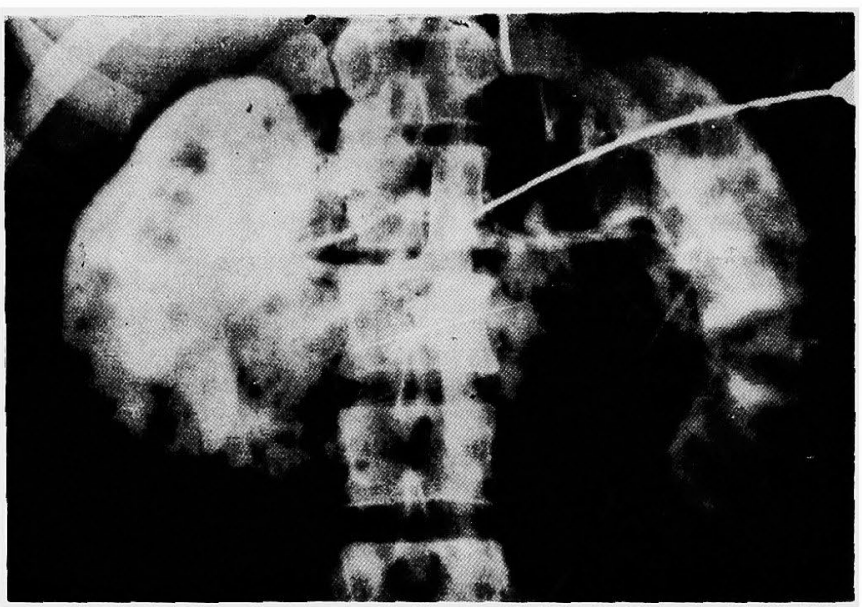

Fig. 8. Congenital hydronephrosis induced by the aberrent artery running from the abdominal aorta to the left kidney.

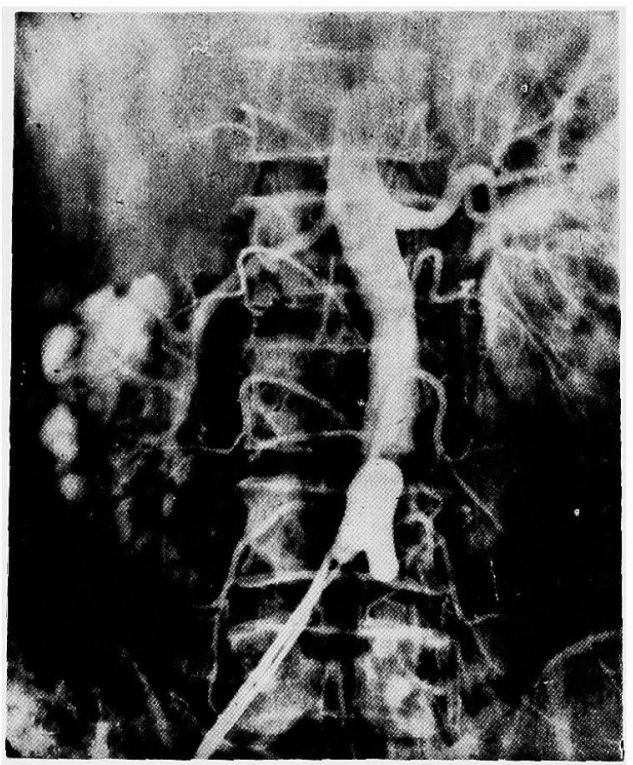

Fig. 9. Right congenital hydronephrosis (examined by employing a balloon eatheter).

hydronephrosis and came to demonstrate in 2 cases with the $x$-ray finding of aberrant vessels (Fig. 8) and to perform nephrectomy in 16 cases where the reduction of the size of the renal arteries and loose intrarenal arterial branches were demonstrated on their renal arteriograms (Fig. 9). 


\section{5) Renal tuberculosis}

Renal tuberculosis shows various pathological pictures, and the changes of a renal arteriogram differ so much according to the type. They may be tentatively classified into an initial and an advanced type. First, there is a question of indication of partial nephrectomy in the lesions, as a progressed chemotherapy is available today. Hereon, a renal arteriogram is of great importance. Because of the findings which it gives, the distribution of the intrarenal arterial branches is seen to become rough in agreement with the lesions, the intrarenal arterial branches running to this portion are observed to be interrupted, and

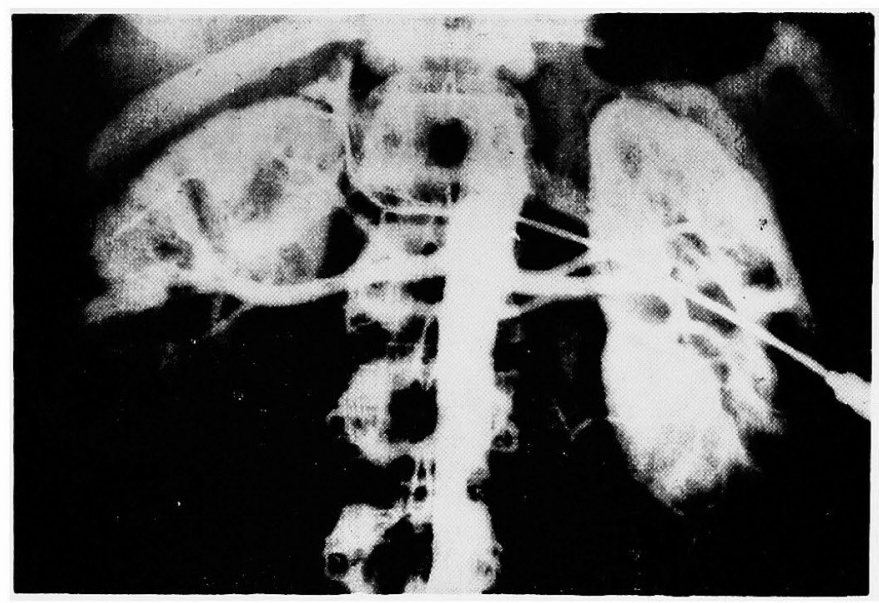

Fig. 10. Right renal tuberculosis. A lesion in the lower pole of the right kidney.

Partial nephrectomy was carried out on the basis of this renal arteriogram.
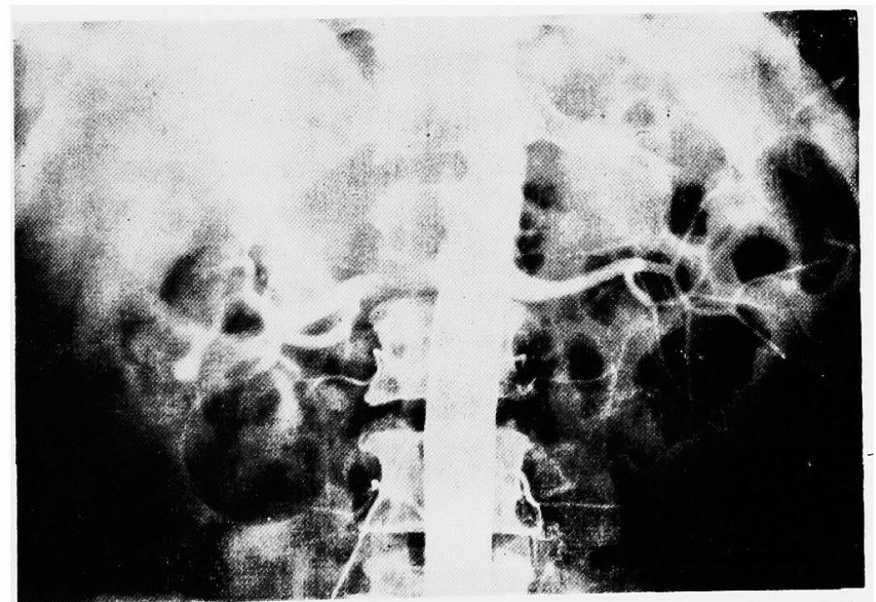

Fig. 11. Left advanced renal tuberculosis (a balloon catheter employed). 
furthermore the nephrogram turns out dim only in this portion (Fig. 10).

We performed partial nephrectomy after deciding the area of the lesions to be resected on the basis of such findings as mentioned above.

If a lesion is an advanced one, the renal artery of any type becomes narrower and the intrarenal arterial branches turn out finer according to the degree of advancement of the lesions (Fig. 11).

As to this relationship between the narrowed caliber of the renal artery and the lowered renal function, Maluf, Ohtani and other investigators pointed out that they were almost in a proportional relation. We made also an observation on the relation between the nephrogram and the caliber of the renal artery on the renal arteriograms obtained in 16 patients who had advanced lesions (Table IV). It

TABLE IV. Aortograms in Renal Tuberculosis

\begin{tabular}{|c|c|c|c|c|c|c|}
\hline \multicolumn{3}{|c|}{ Cases } & \multicolumn{2}{|c|}{ Nephrogram } & \multicolumn{2}{|c|}{ Inner diameter of renal artery } \\
\hline Lname, & sex, & age) & Affected & Healthy & Affected & Healthy \\
\hline M.M. & \% & 43 & + & $H$ & $2.7 \mathrm{~mm}$ & $4.5 \mathrm{~mm}$ \\
\hline I.S. & Q & 42 & + & $t$ & 4.1 & 6.3 \\
\hline I.T. & P & 43 & \pm & \# & 3.6 & 4.5 \\
\hline S.M. & $\delta$ & 21 & + & $H$ & 5.6 & 6.3 \\
\hline M.T. & $\delta$ & 28 & + & + & 4.5 & 6.3 \\
\hline S.N. & 1 & 37 & + & + & 5.4 & 6.3 \\
\hline N.Y. & $\delta$ & 40 & + & $H$ & 5.4 & 6.3 \\
\hline I.A. & $\delta$ & 38 & + & tt & 4.5 & 7.2 \\
\hline K.S. & 우 & 23 & $H$ & H & 3.6 & 6.3 \\
\hline S.K. & 3 & 60 & H & $H$ & 5.4 & 6.3 \\
\hline S.J. & $\hat{b}$ & 43 & + & $H$ & 5.2 & 5.2 \\
\hline O.Y. & 3 & 39 & H & $H$ & 5.4 & 5.4 \\
\hline N.S. & $\mathrm{b}$ & 48 & + & + & 4.5 & 5.4 \\
\hline K.T. & $\delta$ & 43 & + & + & 4.5 & 3.6 \\
\hline I.E. & q & 47 & \pm & H & 2.7 & 4.5 \\
\hline Y.M. & q & 24 & + & H & 2.7 & 5.4 \\
\hline \multicolumn{3}{|l|}{ Total } & \multicolumn{2}{|c|}{16} & 4.4 (average) & 5.7 (average) \\
\hline
\end{tabular}

was shown that in many cases with the renal arteries narrowed, the nephrogram was faint in comparison with the healthy one and the caliber of the renal artery was also fine as compared with the healthy one. The mean value was $4.4 \mathrm{~mm}$ on the affected side, while it was $5.7 \mathrm{~mm}$ on the healthy side. In addition, all these cases were subjected to nephrectomy; the obtained specimens of resection showed serious pathological changes and there were not so many healthy nephrons demonstrated histologically.

As Lattimer et $a l^{7}$ has already stated, the chemotherapeutic agents are transported as far as the lesions by way of blood stream. However, we should promptly resort to nephrectomy because any chemotherapeutic effect cannot be expected in such serious pathological cases as having their renal arteries narrowed 
greatly, the intrarenal arterial branches distributed poorly and the nephrograms visualized faintly or unvisualized.

As seen from the above, the employment of renal arteriography in the cases of renal tuberculosis is of a great clinical significance so that we have so far carried out this radiography in 42 cases in order to obtain from it some hints for determination of the optimum time and method of operation and of the appropriate period of chemotherapy.

\section{6) Unilateral renal hypertension}

This condition is not of a single disease, and may be classified into two major conditions. One is the condition which has pathological changes in the renal artery causing obstruction of the inner lumens of it and then ischemia of the renal parenchyma with the changes diffusing as far as the renal parenchyma. As for the causes of such condition, there are arteriosclerosis, nodal periarteritis, aneurysm and thrombosis of the main renal artery. The other is the condition that leads to unilateral renal impairment. The diseases responsible for this condition are pyelonephritis, nephrolithiasis, hydronephrosis, renal infarct, renal tuberculosis and renal tumor. Renal arteriography is diagnostically indispensable especially to the so-called "Goldblatt-type" hypertension caused by the stenosis of blood vessels. In the disease of this type, according to Abeshouse et $a l .{ }^{8}$ and Inoue, ${ }^{9}$ (1) the renal artery shows complete or incomplete stenosis, or obstruction; and the intrarenal arteial branches are visualized incomplete or obscure. (2) There are alterations in the caliber and walls of the renal artery and the intrarenal arterial branches. In other words, utilization of renal arteriography helps us to decide the principle of treatment of the condition as well as to know what part of the renal artery is stenotic and how far it extends.

We experienced 4 cases of renal arterial stenosis. Of them, 2 cases were due to fibromuscular hyperplasia of the tunica media, one case due to arteriosclerosis and another case due to periarteritis. As to the findings on the renal arteriogram in these cases, stenosis was seen in all cases 2 to $5 \mathrm{~cm}$ from the portion where renal arteries started to branch from the aorta, ranging from $5 \mathrm{~mm}$, to about $3 \mathrm{~cm}$ long, and, moreover, all cases showed the so-called post-stenotic dilatation in succession to the stenotic portions and at the same time the nephrograms were found to be faint and small in size as compared with those of the healthy side. As a result of the observation of the renal arteriograms in these cases, it was shown that if the stenotic area was short in extent, it could be fully visualized by the translumbar method, and that if the area was long in extent, it could not be sufficiently visualized by the translumbar method but distinctly visualized by using a balloon catheter (Figs. 12 and 13).

As for the hypertensive case resulting from the changes in the renal parenchyma we experienced 6 cases which were caused by contracted kidney due 


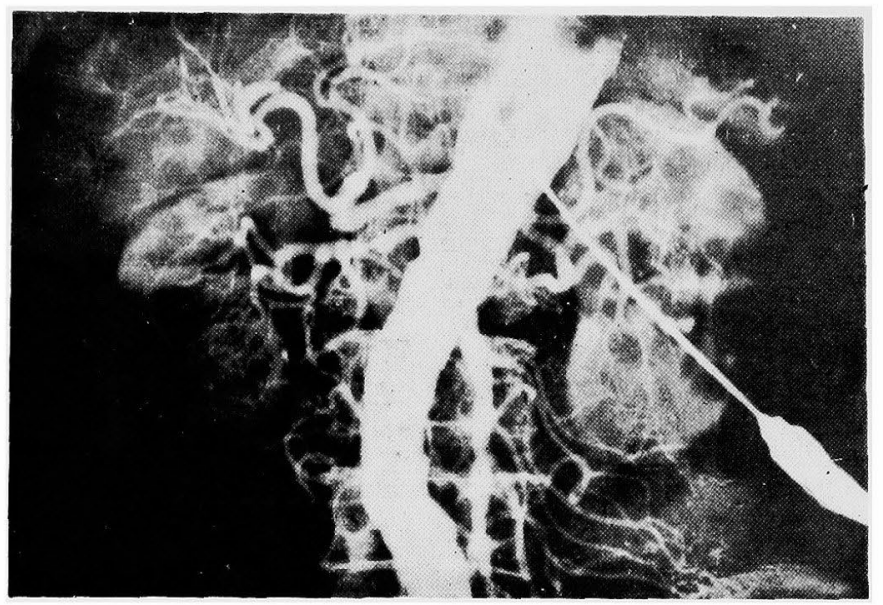

Fig. 12. Unilateral renal hypertension showing right renal arterial stenosis due to arteriosclerosis.

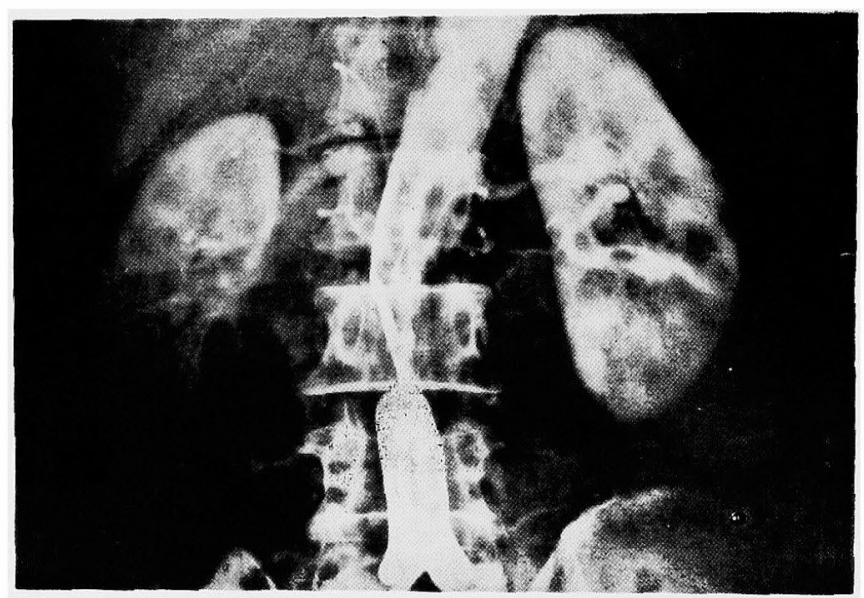

Fig. 13. Unilateral renal hypertension showing right renal arterial stenosis due to periarteritis (examined with a balloon catheter).

to pyelonephritis. Their renal arteriograms revealed narrowed caliber of the renal artery, roughness of the distribution of the intrarenal blood vessels, and the nephrogram obscurer and much less in size than that of the healthy side (Fig. 14).

As mentioned above, when hypertension was proved and there was any difference observed between the right and left kidneys on a pyelogram, we carried out renal arteriography with a suspicion of renal tuberculosis. If the above findings were obtained, the method of treatment could be established at the same time with the radiographic diagnosis. In this sense, significance of this radiographic 


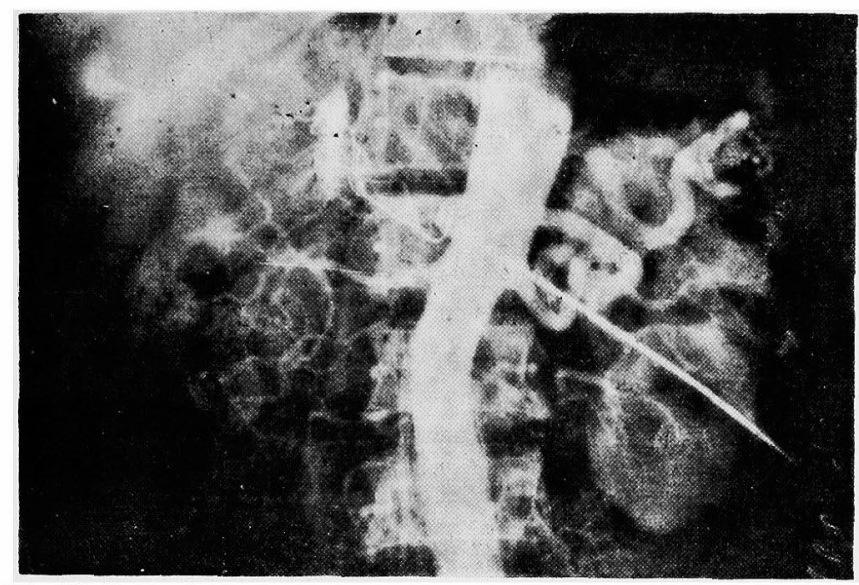

Fig. 14 Unilateral renal hypertension resulting from right contracted kidney due to pyelonephritis.

technique might well be said to be important in the treatment of renal hypertension.

\section{7) Solitary renal cyst}

It is difficult to differentiate this condition form renal neoplasma on a pyelogram. Its diagnosis should be based on a renal arteriogram. In other words, when a case is not seen to show a "pooling" sign on a renal arteriogram and avascularity is proved in agreement with the tumor area, it is diagnosed as solitary renal cyst. We carried out renal arteriography in 3 patients with solitary renal cyst, and succeeded in differentiating it from renal neoplasm. As for
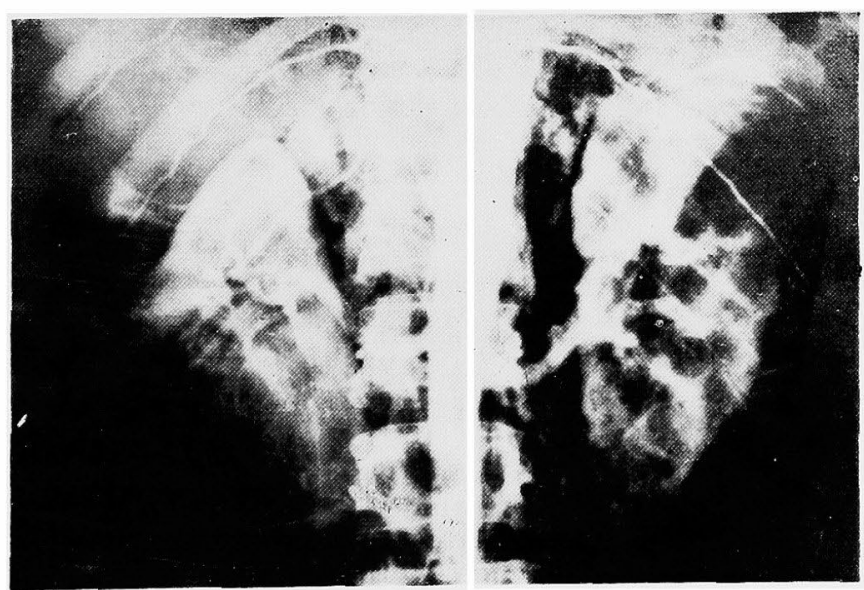

Fig. 15. Right solitary renal eyst with avascularity demonstrated in the lower pole of right kidney. 
the findings in these cases, in addition to the avascularity demonstrated in the area corresponding to the cyst, nephrograms were faint in that area and oppressed intrarenal bood vessels were seen in the marginal portion of the cyst (Fig. 15).

8) Renal neoplasm

Renal arteriography has the highest clinical value in this condition than in any other conditions. Above all, this is the most suitable method in differentiating solitary renal cyst and retroperitoneal tumor. For instance, as already reported by Dos Santos, ${ }^{10}$ there are such signs on $\mathrm{x}$-ray pictures as may be described as "pooling", "laking", "stapling", or "puddling" obtainable in the case of carcinoma of the kidney as a result of contrast-medium retention in renal arteriography. These signs are developed by a number of blood vessels which have been newly and abnormally formed in the tumor and are by no means observable in renal cyst (Fig. 16). All cases of carcinoma of the kidney, however,

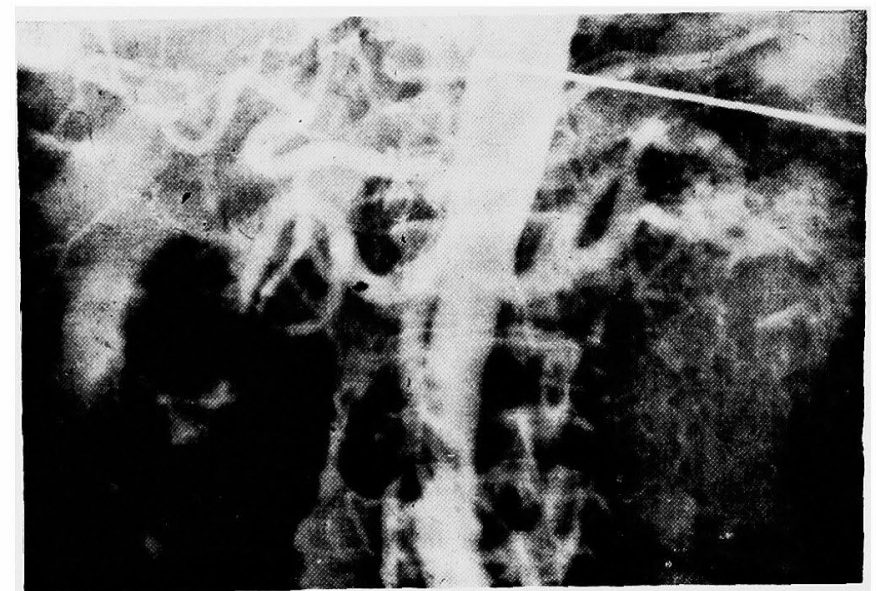

Fig. 16. Carcinoma of left kidney with a "pooling" sign observed in the lower pole of the kidney.

do not show these signs. This phenomenon comes to be out of recognition when a tumor grows very rapidly or when a tumor has progressed so much that its area has become necrosis. On the other hand, a "pooling" picture is sometimes observed in the vascular construction also in the cases other than carcinoma of the kidney. In fact, we obtained the findings similar to the "pooling" sign in a case of neurofibroma affecting a single kidney and a case of tuberculous perinephritis (Figs. 17 and 18). In the case of renal neoplasm, the renal artery is seen to be oppressed by a tumor and to run snake-like or arciform. The abdominal aorta is likewise pushed toward the healthy side and shows an arciform running. In our cases, as seen in Table $\mathrm{V}$ the snake-like running of the renal blood vessels was 


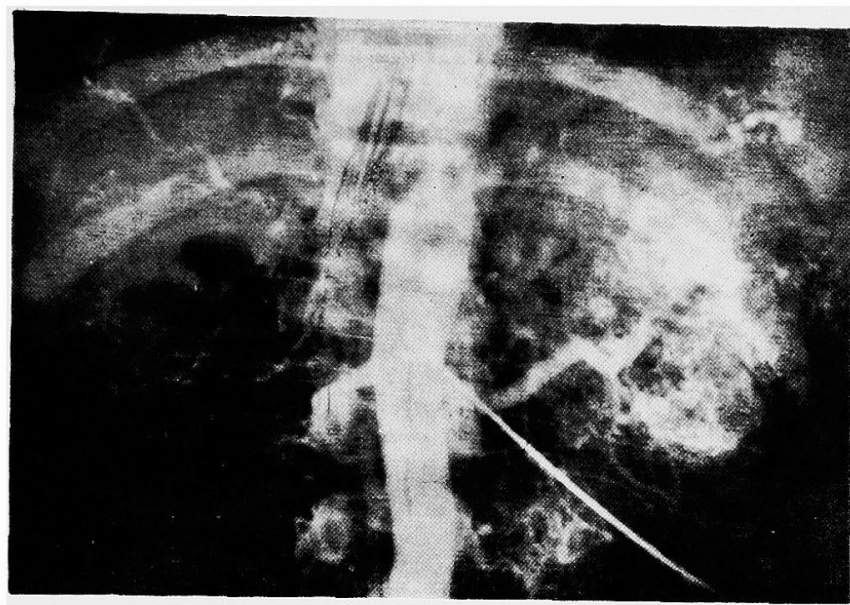

Fig. 17. Neurofibroma in the left single kidney.

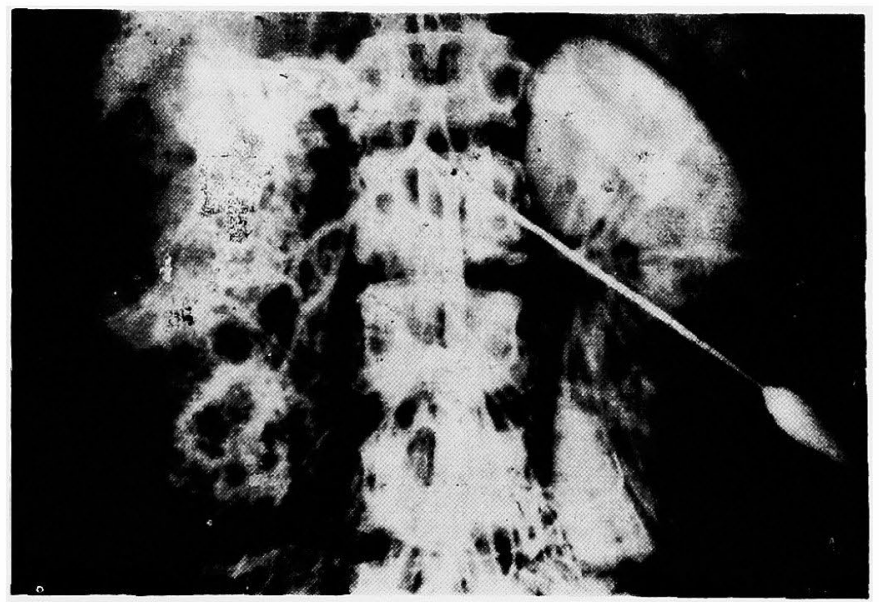

Fig. 18. Perinephritis due to right renal tuberculosis.

seen in 16 out of 17 cases $(94.1 \%$ ) and the arciform running of the abdominal aorta in 13 out of 17 cases $(76.4 \%)$. Besides, the affected renal artery was recognized to be markedly enlarged in some cases. The average size of the renal artery in the 17 cases was $6.5 \mathrm{~mm}$ in diameter on the affected side and $5.6 \mathrm{~mm}$ on the healthy side. This finding almost coincides with the results reported by Ohtani.

Furthermore, renal arteriographic findings help us decide the method of treatment. In other words, it is possible to find a route reaching a kidney and, moreover, to know whether or not any operation is acceptable by examining the size of the area showing a "pooling" sign on a renal arteriogram. We had such 
TABLe V Aortograms in Carcinoma of the Kidney

\begin{tabular}{|c|c|c|c|c|c|}
\hline \multirow[t]{2}{*}{ Cases } & \multirow[t]{2}{*}{ Pooling sign } & \multirow{2}{*}{$\begin{array}{l}\text { Snake-like } \\
\text { renal vessels }\end{array}$} & \multirow{2}{*}{$\begin{array}{l}\text { Arciform of } \\
\text { the abdomi- } \\
\text { nal aorta }\end{array}$} & \multicolumn{2}{|c|}{$\begin{array}{c}\text { Inner diameter of renal } \\
\text { artery }\end{array}$} \\
\hline & & & & Affected & Healthy \\
\hline D.K. & H & + & + & $6.3 \mathrm{~mm}$ & $6.3 \mathrm{~mm}$ \\
\hline K.T. & - & + & - & 6.3 & 6.3 \\
\hline O.S. & + & + & - & 7.2 & 6.3 \\
\hline S.K. & H & + & + & 7.2 & 5.0 \\
\hline T.T. & H & + & + & 5.4 & 5.4 \\
\hline S.S. & - & + & + & 4.5 & 5.4 \\
\hline O.S. & $\mathrm{HH}$ & + & + & 7.2 & 4.5 \\
\hline K.T. & H & t & + & 6.3 & 3.6 \\
\hline S.A. & - & + & - & 5.3 & 5.2 \\
\hline T.K. & - & + & - & 4.5 & 5.6 \\
\hline Y.A. & H & + & + & 7.2 & 6.3 \\
\hline H.T. & H & + & + & 6.8 & 5.2 \\
\hline I.J. & H & + & + & 6.3 & 5.4 \\
\hline S.K. & H & + & + & 8.1 & 7.2 \\
\hline U.B. & - & + & + & 6.2 & 6.2 \\
\hline O.Z. & - & - & + & 6.3 & 5.2 \\
\hline K.I. & H & + & + & 6.3 & 5.6 \\
\hline $\begin{array}{c}\text { Total } \\
17 \text { cases }\end{array}$ & $\begin{array}{l}11 \\
(64.1 \%)\end{array}$ & $\begin{array}{l}16 \\
(94.1 \%)\end{array}$ & $\begin{array}{l}13 \\
(76.4 \%)\end{array}$ & $\begin{array}{c}6.5 \\
\text { (average) }\end{array}$ & $\begin{array}{c}5.6 \\
\text { (average) }\end{array}$ \\
\hline
\end{tabular}

experience in one case. We found that a pooling picture was covering the abdominal aorta, and we reached a conclusion that it was impossible to indicate any proper operation in this case, for infiltration might have already extended as far as the surrounding of the aorta (Fig. 19).

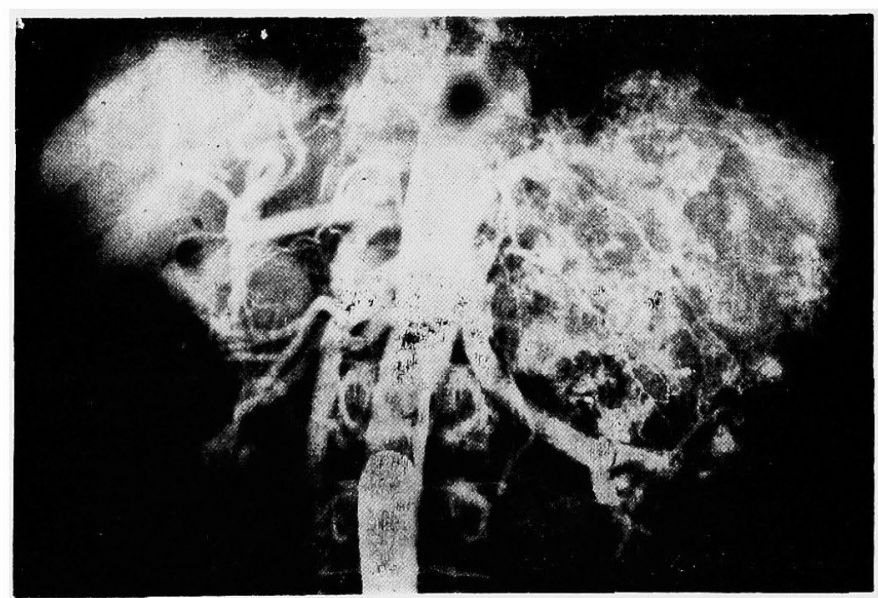

Fig. 19. A case of carcinoma of right kidney diagnosed as inoperable from the renal arteriographic findings (examined with the balloon catheter). 


\section{References}

1) Watanabe, A. Ko-Ken-Shi (Jap.), 1963, 16, 113.

2) Melick, W.F., Yarbrough, C.P. \& Boler, T.D. J. Urol., 1952, 67, 1019.

3) Maluf, N.S.R. \& MoCoy, C.B. Amer. J. Roentgenol., 1955, 73, 533.

4) Ohtani, Y. Acta Urol. Japonica (Jap.), 1960, 6, 1019.

5) Doss, A.K. J. Urol., 1947, 57, 521.

6) Smith, P.G., Rush, T.W. \& Evans, A.T. ibid., 1951, 65, 911.

7) Lattimer, J.K., Hertzberg, A., Herper, J., Berman, M., Bradley, D. \& Veenema, R. ibid., 1952, 67, 750 .

8) Abeshouse, B.S., Scherlis, I., Golden, M. \& Rubin, M. Urol.cutan. Rev., 1951, 55, 517.

9) Inoue, H. Acta Urol. Japonica (Jap.), 1961, 7, 157.

10) Dos Santos, R. Arch. Mal. Reins., 1934, 8, 313. 